\title{
Autonomia da gestante na escolha do tipo de parto*
}

\author{
Autonomy of the pregnant woman in the selection of the type of childbirth \\ Autonomía de la gestante en la selección del tipo de parto
}

Mônica Maria de Jesus Silva a

Universidade de São Paulo, Brasil

monicamjs@usp.br

ORCID: http://orcid.org/0000-0002-4532-3992

Semara Carollini Brandão Silva

Faculdades Integradas Asmec, Brasil

ORCID: http://orcid.org/0000-0002-1066-6709

Gabriel Arruda Melo

Faculdades Integradas Asmec, Brasil

ORCID: http://orcid.org/0000-0003-2996-9694
DOI: https://doi.org/10.11144/Javeriana.ie21-2.aget

Recepção: 10 Agosto 2017

Aprovação: 03 Setembro 2018

Publicado: 30 Novembro 2019

\section{Resumo:}

Introdução: inúmeros fatores estão envolvidos na tomada de decisão da mulher pelo tipo de parto a ser escolhido. Quando se trata da mulher primigesta, as experiências prévias não se fazem presentes, porém, outros fatores podem então influenciá-la na escolha pelo modo de parir. Objetivo: identificar a preferência da gestante primigesta quanto à via de parto, conhecer os fatores que influenciam a sua tomada de decisão nessa escolha, e as suas expectativas em relação ao parto mediante a via escolhida. Método: estudo descritivo com abordagem quantitativa realizado com 35 primigestas em acompanhamento pré-natal nas unidades públicas de saúde em um município do Sul de Minas Gerais, Brasil. A coleta de dados ocorreu em agosto e setembro de 2015 e utilizou um formulário semiestruturado. Resultados: predominou a escolha pela via de parto vaginal, a presença de expectativas positivas em relação a via escolhida e a não influência de terceiros sobre a tomada de decisão. Conclusão: a decisão da mulher na escolha da via de parto está se transformando em uma preferência e não apenas em uma indicação profissional, o que ressalta a importância da educação em saúde na consulta pré-natal.

Palavras-chave: enfermagem, parto, parto normal, cesárea, preferência do paciente.

\section{Abstract:}

Introduction: Innumerable factors are considered in the decision-making when choosing the type of childbirth. When it is a firsttime woman, there are not previous experiences, so other factors can influence her in the selection of how to give birth. Objective: to identify the preference of the first-time pregnant woman in terms of the type of childbirth, to know the factors that influence her decision-making in that selection and her expectations in relation to childbirth through the chosen type of birth-delivery. Method: A descriptive study with a quantitative approach carried out with 35 first-timers in prenatal care in public health units in a municipality in the south of Minas Gerais, Brazil. Data collection was conducted in August and September 2015 and a semistructured interview was used. Results: the selection of the vaginal delivery, the presence of positive expectations in relation to the chosen way of birth-giving and the lack of influence of third parties on the decision-making process prevailed. Conclusions: The decision of the women in selecting the way of birth- delivery is turning in a preference, but a simply a professional indication, which highlights the importance of health education in prenatal consultation.

Keywords: nursing, delivery, normal delivery, caesarean section, patient preference.

\section{Resumen:}

Introducción: Innumerables factores están involucrados en la toma de decisiones de la mujer por el tipo de parto a ser escogido. Cuando se trata de una mujer primeriza, las experiencias previas no se hacen presentes, por tanto, otros factores pueden influenciarla en la selección del modo de dar a luz. Objetivo: identificar la preferencia de la gestante primeriza en cuanto a la forma de parto, conocer los factores que influencian su toma de decisión en esa selección y sus expectativas en relación al parto mediante la vía

\section{Autor notes}

\footnotetext{
a Autora de correspondência. Correio eletrônico: monicamjs@usp.br
} 
escogida. Método: estudio descriptivo con abordaje cuantitativo realizado con 35 primerizas en acompañamiento prenatal en las unidades públicas de salud en un municipio del Sur de Minas Gerais, Brasil. La recolección de datos se realizó en agosto y septiembre de 2015 y utilizó una entrevista semiestructurada. Resultados: predominó la selección de la vía de parto vaginal, la presencia de expectativas positivas en relación a la vía escogida y la no influencia de terceros sobre la toma de la decisión. Conclusiones: la decisión de las mujeres en la selección de la vía del parto se está transformando en una preferencia y no simplemente en una indicación profesional, lo que resalta la importancia de la educación en salud en la consulta prenatal.

Palabras clave: enfermería, parto, parto normal, cesárea, preferencia del paciente.

\section{Introdução}

O processo parir é permeado por aspectos sociais e culturais que influenciam diretamente na preferência da mulher por determinado tipo de parto. Inúmeros fatores estão envolvidos no processo de tomada de decisão da mulher pelo tipo de parto a ser escolhido, os quais perpassam por experiências anteriores, informações concebidas ao longo da vida, inclusive durante a assistência pré-natal, além de outros mecanismos individuais, coletivos e institucionais (1).

Quando se trata da mulher primigesta que vivência pela primeira vez uma gestação, as experiências prévias não se fazem presentes e outros fatores podem então influenciá-la na escolha pelo modo de parir.

No entanto, o respeito às escolhas da mulher deve preponderar para que o nascimento seja um momento ímpar, no qual ela tem o direito de participar das decisões sobre sua saúde e ações relacionadas ao seu próprio corpo, inclusive o tipo de parto ao qual será submetida (1), conforme preconiza o Programa de Humanização no Pré-Natal e Nascimento (PHPN), instituído no ano 2000 pelo Ministério da Saúde do Brasil, visando a humanização da assistência (2).

Neste contexto, considerando a importância da autonomia da mulher na escolha da via de parto para uma tomada de decisão de forma crítica e segura, inserida no contexto do empoderamento feminino no processo de parir, este estudo teve como objetivos identificar a preferência da gestante primigesta quanto à via de parto, conhecer os fatores que influenciam a sua tomada de decisão nessa escolha, assim como suas expectativas em relação ao parto mediante a via de parto escolhida.

\section{Método}

Estudo descritivo com abordagem quantitativa realizado no período de agosto a setembro de 2015 com gestantes primigestas acompanhadas em três unidades do Programa de Saúde da Família que oferecem atendimento pré-natal, no âmbito do Sistema Único de Saúde, de um município do Sul de Minas Gerais, Brasil.

Como critérios de inclusão para o estudo, adotou-se ser gestante; estar grávida pela primeira vez, idade maior ou igual a 18 anos, estar em acompanhamento pré-natal natal nas referidas unidades de saúde. Os critérios de exclusão foram a presença de condição clínica que represente uma indicação para o parto cesáreo e a incapacidade de responder ao instrumento de coleta de dados sem a participação de outra pessoa.

Para a definição da amostra, realizou-se o levantamento do número de gestantes primigestas em acompanhamento pré-natal nas unidades de saúde no ano anterior. Desta forma, a mostra foi composta por 35 gestantes primigestas, selecionadas por meio de uma amostragem por conveniência. Ressalta-se que, no período em estudo, todas as gestantes primigestas abordadas aceitaram participar do estudo. Foram excluídas quinze gestantes por se tratarem de menores de 18 anos e não houve perdas.

Para a coleta de dados foi utilizado um formulário semiestruturado, de autoria dos pesquisadores, contendo 24 perguntas abertas e fechadas referentes a questões obstétricas, sócio demográficas e concernentes à preferência da via de parto, expectativa em relação a via escolhida e fatores de influência na tomada de decisão. 
O formulário foi submetido a um teste piloto antes da coleta de dados em uma amostra de gestantes, que foi descartada da amostra final, com o objetivo de garantir a compreensão pelas participantes.

$\mathrm{Na}$ coleta de dados, o formulário foi aplicado pelos pesquisadores por meio de entrevista. A abordagem das gestantes ocorreu na sala de espera das unidades de saúde, enquanto as gestantes aguardavam a consulta pré-natal.

Os dados coletados foram organizados e armazenados em uma planilha no programa Microsoft Excel'. Em seguida, os mesmos foram analisados no programa Statisical Package for Social Sciencies (SPSS), versão 20.0. As variáveis categóricas foram descritas em números absolutos e percentuais.

O estudo foi aprovado pelo Comitê de Ética em Pesquisa das Faculdades Integradas Asmec-União das Instituições de Serviço Ensino e Pesquisa, sob parecer no 1129105 . A pesquisa foi conduzida cumprindo as Diretrizes e Normas Regulamentadoras de Pesquisa em Seres Humanos do Conselho Nacional de Saúde, Resolução 466/2012.

\section{Resultados}

Quanto a caracterização da amostra, a faixa etária variou de 18 a 40 anos de idade, com média de 26 anos e maior frequência na faixa entre 26 e 30 anos. A distribuição conforme a ocupação ou profissão apresentou maior quantidade de mulheres que tinha alguma ocupação ou profissão remunerada. Predominaram ainda as gestantes casadas, que frequentaram a escola até o ensino médio e católicas. Quanto à renda familiar mensal, esta variou de 780 reais a 4 mil reais, com média de 1854 reais e maior frequência de dois a três salários mínimos. A caracterização da amostra é apresentada na tabela 1.

TABELA 1.

Caracterização socioeconômica e demográfica das gestantes. Monte Sião, MG, Brasil, 2015

\begin{tabular}{|c|c|c|}
\hline Variáveis & $\mathrm{F}$ & $\%$ \\
\hline \multicolumn{3}{|l|}{ Faixa Etária (anos) } \\
\hline Abaixo de 20 & 05 & 11,4 \\
\hline $20-25$ & 11 & 28,5 \\
\hline $26-30$ & 09 & 37,1 \\
\hline $31-35$ & 09 & 20,0 \\
\hline Acima de 35 & 01 & 3,0 \\
\hline \multicolumn{3}{|l|}{ Estado civil } \\
\hline Casada & 30 & 85,7 \\
\hline Solteira & 05 & 14,3 \\
\hline \multicolumn{3}{|l|}{ Escolaridade } \\
\hline Fundamental incompleto & 02 & 6,0 \\
\hline Fundamental completo & 06 & 17,0 \\
\hline Médio incompleto & 09 & 25,3 \\
\hline Médio completo & 16 & 45,7 \\
\hline Superior incompleto & 01 & 3,0 \\
\hline Superior completo & 01 & 3,0 \\
\hline \multicolumn{3}{|l|}{ Religiẫo } \\
\hline Católica & 20 & 56,9 \\
\hline Evangélica & 13 & 37,1 \\
\hline Sem crença & 02 & 6,0 \\
\hline \multicolumn{3}{|c|}{ Renda Familiar Mensal (salários mínimos)* } \\
\hline Até 1 & 01 & 3,0 \\
\hline Acima de 1 até 2 & 07 & 20,0 \\
\hline Acima de 2 até 3 & 10 & 28,5 \\
\hline Acima de 3 & 03 & 9,0 \\
\hline Não informado & 14 & 39,5 \\
\hline \multicolumn{3}{|l|}{ Ocupação } \\
\hline Dolar & 07 & 20,0 \\
\hline Exerce atividade trabalhista remunerada & 27 & 77,0 \\
\hline Estudante & 01 & 3,0 \\
\hline
\end{tabular}

F: frequência absoluta; \%: frequência em porcentagem; * salário mínimo vigente de 788,00 reais. Fonte: dados da pesquisa 
Considerando as semanas de gestação em que se encontravam as participantes, predominaram as que estavam no segundo trimestre gestacional, conforme evidenciado na tabela 2.

TABELA 2.

Idade gestacional das gestantes, segundo o trimestre, em atendimento prénatal nas unidades do Programa Saúde da Família. Monte Sião, MG, Brasil, 2015

\begin{tabular}{lcc}
\hline \multicolumn{1}{c}{ Variáveis } & F & \% \\
\hline Idade gestacional & & \\
\hline $1^{\circ}$ trimestre & 7 & 20,0 \\
\hline $2^{\circ}$ trimestre & 15 & 42,8 \\
\hline $3^{\circ}$ trimestre & 13 & 37,2 \\
\hline
\end{tabular}

F: frequência absoluta; \%: frequência em porcentagem. Fonte: dados da pesquisa

No que se refere às informações sobre o parto, todas as gestantes relataram não ter dúvidas e a maior parte informou ser o médico a principal fonte de informação sobre a gravidez e o parto. Além disso, a maioria das gestantes recebeu informações durante o pré-natal sobre os tipos de parto e relataram que foram informadas que tanto o parto vaginal como o parto cesariano são seguros (tabela 3 ).

TABELA 3.

Distribuição das participantes segundo informações sobre o parto. Monte Sião, MG, Brasil, 2015

\begin{tabular}{|c|c|c|}
\hline Variáveis & $\mathrm{F}$ & $\%$ \\
\hline \multicolumn{3}{|c|}{ Dúvidas sobre o tipo de parto } \\
\hline Não & 35 & 100 \\
\hline \multicolumn{3}{|c|}{ Fonte de informação sobre gestação e part } \\
\hline Médico & 16 & 45,7 \\
\hline Internet & 07 & 20,0 \\
\hline Amigos & 04 & 12,0 \\
\hline Família & 05 & 13,3 \\
\hline Enfermeiro & 01 & 3,0 \\
\hline Escola & 02 & 6,0 \\
\hline \multicolumn{3}{|c|}{ Orientação no pré-natal } \\
\hline Sim & 20 & 57,1 \\
\hline Não & 15 & 42,9 \\
\hline \multicolumn{3}{|c|}{ Orientação recebida } \\
\hline Parto vaginal é mais seguro & 03 & 9,0 \\
\hline Parto cesariana é mais seguro & 01 & 3,0 \\
\hline Ambos são seguros & 17 & 48,0 \\
\hline Não houve esclarecido & 14 & 40,0 \\
\hline
\end{tabular}

Outro dado que emerge do presente estudo é o número de consultas realizadas no pré-natal. Entre as gestantes, $45,7 \%$ (16) realizaram de 2 a 4 consultas; $31,5 \%$ (11) participaram de cinco a sete consultas e 22,8 \% estiveram presentes em mais de sete consultas do acompanhamento pré-natal.

Acerca da preferência pelo tipo de parto e tomada de decisão, $88,6 \%$ das participantes possuíam uma via de parto de preferência no momento da entrevista. Entre estas, 83,9\% (26) optaram inicialmente pelo 
parto vaginal. Entre os motivos para a escolha do parto vaginal, 69,2\% (18) das gestantes referiu a rápida recuperação pós-parto; seguida por 15,6\% (4) das participantes que escolheram este tipo de parto porque ele é natural. Também foram mencionados como motivo para escolha do parto vaginal a dor única (3,8\%), a informação de que cesárea é prejudicial ao bebê $(3,8 \%)$, o desejo de evitar complicações no pós-operatório $(3,8 \%)$ e a concepção de que o parto vaginal é o melhor momento para o nascimento $(3,8 \%)$.

Já entre as participantes que manifestaram preferência pelo parto cesariana, a indicação clínica e o medo da dor, estão entre os principais motivos que a levaram a optar pelo parto cirúrgico, sendo referidos por $40 \%$ delas. A recomendação médica foi mencionada por $20 \%$ destas (tabela 4 ).

TABELA 4.

Distribuição das participantes segundo à escolha da via de parto. Monte Sião, MG, Brasil, 2015

\begin{tabular}{lccc}
\hline Variáveis & F & $\%$ \\
\hline \multicolumn{1}{c}{ Escolha da vida de parto } & & \\
\hline Sim & 31 & 88,6 \\
\hline Não & 04 & 11,4 \\
\hline \multicolumn{2}{c}{ Influência sobre escolha } \\
\hline Sim & 10 & 28,5 \\
\hline Não & 25 & 71,5 \\
\hline \multicolumn{2}{c}{ Escolha final } & & \\
\hline Parto vaginal por escolha da mulher & 01 & 2,8 \\
\hline Parto cesariana por escolha do profissional & 03 & 8,5 \\
\hline Parto cesariana por escolha de ambos & 04 & 11,4 \\
\hline Não possui decisão final & 27 & 77,3 \\
\hline
\end{tabular}

F: frequência absoluta; \%: frequência em porcentagem. Fonte: dados da pesquisa

Quanto à tomada de decisão pela via de parto, a maioria das participantes do estudo $(71,4 \%)$, referiu não ter sofrido influência. No entanto, o médico foi apontado como o principal fator de influência (60\%) entre as gestantes que referiram ter sido influenciadas na escolha, seguido pela família (30\%) e amigos (10\%).

Além disso, apesar da grande maioria das participantes apresentar preferência por alguma via de parto, $77,3 \%$ das gestantes relataram não ter uma decisão concreta quanto à escolha final da via para a realização de seu parto, seguidas de $11,4 \%$ que informaram ter escolhido o parto cesariana por decisão compartilhada entre ela e o médico, como descrito na tabela 4.

$\mathrm{Na}$ amostra em estudo, a maioria das gestantes possuíam expectativa positiva em relação à via de parto escolhida e apenas uma gestante afirmou ter expectativa negativa em relação ao tipo de parto escolhido.

\section{Discussão}

O predomínio de gestantes na faixa etária entre 20 e 30 anos revela similaridade com estudo realizado em Campo Grande, MS, Brasil (1). A discorrer sobre a relação entre idade materna e via de parto, outro estudo brasileiro, realizado em Santa Catarina, demonstrou que a idade das gestantes não influenciou significativamente na escolha da via de parto. No entanto, a preferência pelo parto natural parece ter relação diretamente proporcional à idade materna, traduzindo-se em uma reflexão sobre as consequências das vias de parto em consequência do amadurecimento da mulher (3).

Em análise do estado civil das participantes, observou-se maior frequência de gestantes casadas. Esses dados corroboram com os dados encontrados em pesquisa realizada em Florianópolis, Brasil (4).

No tocante à escolaridade, constatou-se a presença de um perfil de médio nível de instrução, considerando que a maioria das participantes possuem ensino médio completo. 
Ao relacionar a escolaridade e a escolha da via de parto, estudo realizado em Santa Catarina, Brasil, relatou maior ocorrência de preferência pelo parto natural entre mulheres com maior nível educacional, portadoras de ensino médio incompleto e superior completo (3). Como justificativa desta escolha, destaca-se que o maior nível de escolaridade possa estar atrelado à maior informação sobre os benefícios do parto natural para mãe e filho.

No que condiz à renda familiar, verificou-se uma variação de 1 a 4 salários mínimos com predomínio de renda acima de 2 até 3 salários. Ao discorrer acerca da renda familiar, outro estudo apontou para a relação entre preferência por cesárea e o maior nível econômico, isso, talvez, porque a maioria dessas mulheres realizaram o pré-natal na rede privada e que, tal fato associado a motivos diversos, culminaram na escolha da cesariana (5).

Em relação à ocupação, a maioria das gestantes exercem atividade trabalhista remunerada. Tal resultado demonstra uma mudança nas características das mulheres contemporâneas, sendo semelhante ao encontrado em outro estudo que apontou 80\% (4) das mulheres possuíam ocupação ou trabalho remunerado.

No presente estudo, a maioria das gestantes informou professar a religião católica. Dado semelhante foi encontrados em estudo desenvolvido em um município no Estado do Mato Grosso do Sul (6).

No que tange às variáveis sobre o parto, quando perguntadas sobre a existência de dúvidas sobre os tipos de parto, todas as gestantes relataram não as ter.

O esclarecimento de dúvidas, assim como o fortalecimento dos conhecimentos da gestante, são ações a serem desenvolvidas como estratégias de trabalho no acompanhamento pré-natal que permitem a integração de profissionais e gestantes, constituindo um momento de acolhida, escuta, vínculo, compartilhamento de experiências e trocas mútuas (7).

Já no que condiz à principal fonte de informação sobre a gravidez e o parto, a maior parte das gestantes (45,71\%) indicou o médico. As pessoas que constituem o círculo social da gestante, quer sejam a família, amigos e o parceiro, são essenciais para desenvolvimento de uma gravidez saudável, uma vez que a gestante precisa de pessoas de confiança para dividir seus medos, descobertas e alegrias (8). Na escolha da via de parto, as informações fornecidas por estas pessoas, assim como pelos profissionais de saúde podem interferir de modo favorável ou não, considerando-se a qualidade da orientação e a sua influência (3).

Considerando que a cada tipo de parto relaciona-se riscos e benefícios, a construção de um posicionamento da mulher acerca dos tipos de parto é importante para que ela possa requerer o que é melhor para a sua saúde e de seu filho (9).

Neste contexto, o preparo para o parto deve acontecer desde o pré-natal. Momento em que o direito de tomada de decisão sobre qual tipo de parto deve ser assegurado pelo profissional de saúde à mulher e expostas as vantagens, benefícios, desvantagens, bem como as possíveis intercorrências na realização de cada tipo de parto (10). Estas ações contribuem e embasam a humanização da assistência conforme preconiza o Programa de Humanização no Pré-Natal e Nascimento (PHPN), instituído no ano 2000 pelo Ministério da Saúde, visando maior acesso e qualidade do acompanhamento de gestantes e recém-nascidos e contribuindo para a redução das taxas de mortalidade materna e neonatal (9).

Desta forma, no que se refere ao recebimento de orientações sobre os tipos de parto durante o pré-natal, observou-se que a maioria das participantes recebeu informações. Este é sinal de que os profissionais da saúde aderiram à Política de Humanização do parto, como recomendado pelo Ministério da Saúde do Brasil.

Contrariamente ao presente estudo, a literatura demonstra também resultados diferentes, os quais apontaram a baixa taxa de orientação sobre os tipos de parto (7), suas vantagens e desvantagens com a subutilização do pré-natal como espaço educativo.

Entre as gestantes, a maioria $(45,7 \%)$ realizou de 2 à 4 consultas de pré-natal. De acordo com o Ministério da Saúde do Brasil, é preconizado que as consultas durante o pré-natal sejam iniciadas precocemente, logo no primeiro trimestre e realizadas no mínimo seis consultas preferencialmente, uma no primeiro trimestre, duas no segundo trimestre e três no último trimestre (11). 
Considerando as semanas de gestação em que se encontravam as participantes, o número de consultas realizadas está dentro dos parâmetros recomendados e os resultados estão em consonância com o aumento do número de consultas por gestantes e da cobertura pré-natal apresentado no Brasil nos últimos anos. O maior número de consultas permite maior oportunidade de promoção de saúde e transmissão de conhecimento à gestante, e consequentemente mais informações sobre o parto (11).

Em análise as orientações recebidas acerca da segurança de cada via de parto, prevaleceu a informação de que ambas as vias de parto são seguras. O Ministério da Saúde do Brasil enfatiza que o parto normal é o mais seguro e benéfico para a mãe e o bebê. Já o parto cesariano é a via de maior exposição da mulher e de seu filho à riscos, entre os quais estão nascimentos prematuros, mortes perinatais e maternas, bem como infecções (10).

Sobre a preferência pelo tipo de parto, a maioria das gestantes optou inicialmente pelo parto vaginal, contrariando as estatísticas crescentes de opção pela cesárea no Brasil, demonstrando a dissonância existente, muitas vezes, entre o desejo da mulher e a realidade vivida no momento do parto.

Este resultado, está em conformidade com outra pesquisa em que $75 \%$ das participantes também relataram preferir o parto vaginal (9), confrontando a ideia de que a cultura da cesárea está arraigada em nossa sociedade e indicando que apesar da cesariana apresentar um perfil crescente no país, o anseio pelo parto vaginal ainda prevalece entre as mulheres.

Entre as participantes que preferem o parto vaginal, a recuperação mais rápida foi o principal motivo apontado pelas participantes. Resultado já evidenciado em outro estudo que demonstrou que a escolha se embasou na preferência pessoal ou familiar e na experiência anterior, correspondendo a $100 \%$, o que sugere que fatores familiares, socioculturais, assim como a experiência anterior são preponderantes na predileção por esse tipo de parto.

Já entre as participantes que optaram pela cesariana, a predileção foi amparada no medo de sentir dor durante o parto vaginal, indicação clínica e recomendações médicas. O medo do parto vaginal também foi fator contribuinte pela escolha do parto cesárea já evidenciado pela literatura (9). Desta forma, mulheres que vivenciavam a dor do parto como sendo algo natural do processo de parir fizeram opção pelo parto vaginal.

No que se refere à dor, a equipe de enfermagem tem um papel preponderante, uma vez que através de seu cuidado, situações sobre a percepções dolorosas das mulheres são reveladas. Dessa forma, tão importante quanto estar atento à escala de dor, é também indagar a mulher sobre suas expectativas, dúvidas e temores sobre a experiência que se inicia (12).

No que se refere à influência na tomada de decisão na escolha da via de parto, os achados observados no presente estudo se assemelham aos descritos em outra pesquisa na qual entre as gestantes que preferiram o parto cesárea, a indicação do médico e o medo do parto normal foram frequentes, $30 \% \mathrm{em}$ ambas variáveis (9).

No processo decisório sobre a via de parto, muitas vezes, a vulnerabilidade da mulher, somada à detenção do conhecimento pelo médico podem favorecer a construção de uma relação permeada por certa passividade da gestante frente ao médico durante o pré-natal, na qual a gestante valorizaria mais a opinião do profissional de saúde em detrimento da sua (3).

Neste contexto, a decisão médica, tida como supostamente indiscutível pela mulher, prevalece na escolha da via de parto, a qual ocorre independente das compreensões e decisões estabelecidas no âmbito familiar, com pouca participação da gestante, na maioria das vezes (9).

Quando indagadas sobre a escolha final da via para a realização de seu parto e a interferência médica nessa escolha, apesar da grande maioria das participantes apresentar preferência por alguma via, a maior parte delas ainda não tinha uma decisão final.

A decisão do profissional de saúde tem um peso significativo na decisão da mulher pela via do parto, uma vez que o pré-natal se configura com um espeço privilegiado de contato entre o profissional e a mulher para esclarecimentos e informações sem, contudo, desqualificar as crenças da gestante acerca das vias de parto. 
Este estudo evidenciou ainda a presença de expectativas positivas em relação a via parto escolhida, entre a maioria das gestantes primigestas. Este resultado contrasta com outro estudo no qual a expectativa das gestantes em relação ao parto era negativa uma vez que fundamentava-se no medo e na ansiedade (8).

A expectativa da mulher quanto ao tipo de parto é consequência do modo como as informações são disponibilizadas a ela e de como estas são interpretadas conforme o seu meio sociocultural.

Assim, o desenvolvimento de atividades educativas é de suma importância em todo o ciclo gravídicopuerperal, e ainda mais no acompanhamento pré-natal, quando a gestante deve ser bem orientada para que possa viver o parto de maneira positiva.

Neste estudo encontraram-se resultados relevantes referentes a escolha da via de parto, verificando-se que as gestantes grávidas pela primeira vez, recebem muitas influências que interferem em suas decisões. $\mathrm{O}$ fator limitante foi o pequeno número de gestantes.

\section{Conclusão}

O paradoxo existente entre o aumento da gradativo da conscientização da mulher acerca dos benefícios do parto vaginal e o alto índice de cesarianas no Brasil demonstra uma lacuna existente no processo de orientação e preparo da mulher para sua decisão, a qual a impede de assumir o protagonismo na escolha do parto desejado por meio de uma pseudoautonomia que a induz a uma escolha que nem sempre é a mais benéfica ou até mesmo desnecessária e que, assim, justifica a atual cultura da cesárea que instituiu-se no sistema de saúde brasileiro.

Assim, diante deste cenário, observa-se que apesar da escolha pelo tipo de parto, muitas vezes, ser influenciada, carente de conhecimento e orientação que acabam por diminuir a autonomia da gestante na tomada de decisão, a mesma está se transformando em uma preferência da mulher e não apenas em uma indicação profissional.

Neste contexto, infere-se a importância da educação em saúde orientada pelos profissionais durante o pré-natal, informando as vantagens e desvantagens dos diferentes tipos de parto, com vistas a resgatar o protagonismo da mulher no processo de nascimento, oportunizando a ela decidir sobre o tipo de parto que deseja ter de forma crítica e reflexiva, com base em informações consistentes e evidências científicas. Informadas, as mulheres poderão ser multiplicadoras e incentivadoras do parto vaginal, estimulado pela Organização Mundial da Saúde (OMS) e Ministério da Saúde do Brasil.

\section{Referências}

1. Nascimento RRP, Arantes SL, Souza EDC, Contrera L, Sales APA. Escolha do tipo de parto: fatores relatados por puérpera. Rev Gaúcha Enferm. 2015;36(esp):119-26. https://dx.doi.org/10.1590/1983-1447.2015.esp.56496

2. Ministério da Saúde. Programa de humanização do parto: Humanização no pré-natal e nascimento. Brasília: Ministério da Saúde; 2002.

3. Junior TL, Steffani JA, Bonamigo EL. Escolha da via de parto: expectativa de gestantes e obstetras. Rev Bioét. 2013;21(3):509-17.

4. Velho MB, Santos EKA, Collaço VS. Parto normal e cesárea: representações sociais de mulheres que os vivenciaram. Rev Bras Enferm. 2014;67(2): 282-9. https://dx.doi.org/10.5935/0034-7167.20140038

5. Copelli FHS, Rocha L, Zampieri MFM, Gregório VRP, Custódio ZAO. Fatores determinantes para a preferência da mulher pela cesariana. Texto Contexto Enferm, Florianópolis. 2015;24(2): 336-43. https://dx.doi.org/10.1 590/0104-07072015000430014

6. Rezende CL, Souza JC. Qualidade de vida das gestantes de alto risco de um centro de atendimento à mulher. Psicol Inf. 2012;16(16):45-69. 
7. Anversa ETR, Bastos GAN, Nunes LN, Dal Pizzol TS. Qualidade do processo da assistência pré-natal: unidades básicas de saúde e unidades de Estratégia Saúde da Família em município no Sul do Brasil. Cad Saúde Pública (Rio de Janeiro). 2012;28(4):789-800. https://dx.doi.org/10.1590/S0102-311X2012000400018

8. Ferreira LA, Silva JAJ, Zuffi FB, Mauzalto ACM, Leite CP, Nunes JS. Expectation of pregnant women in relation to childbirth. R Pesq: Cuid Fundam Online. 2013;5(2):3692-97. https://dx.doi.org/10.9789/2175-5361.2013 $. v 5 \mathrm{i} 2.3692-3697$

9. Weidle WG, Medeiros CRG, Grave MTQ, Dal Bosco SM. Escolha da via de parto pela mulher: autonomia ou indução? Cad Saúde Colet. 2014;22(1):46-53. https://dx.doi.org/10.1590/1414-462X201400010008

10. Ministério da Saúde (BR). Secretaria de Atenção à Saúde. Departamento de Atenção Básica. Atenção ao pré-natal de baixo risco. Brasília (DF); 2012.

11. Ministério da Saúde (BR). Secretaria de Políticas de Saúde. Assistência pré-natal: manual técnico. Brasília (DF); 2000.

12. Montoya DIG, Mazuelo EMG, López CPH. Experiencias de las mujeres durante el trabajo del parto y parto. Av Enferm. 2015;33(2):271-81. https://doi.org/10.15446/av.enferm.v33n2.42279

\section{Notas}

* Artigo de pesquisa Artigo extraído do trabalho de conclusão de curso intitulado: A autonomia da gestante primigesta na escolha da via de parto: uma análise dos fatores de influência e de suas expectativas.

1. Financiamento: esta pesquisa foi financiada com investimento próprio dos autores.

2. Conflito de interesse: não há conflito de interesse.

\section{Licencia Creative Commons CC BY 4.0}

Como citar este artigo: Silva MM de J, Silva SCB, Melo GA. Autonomia da gestante na escolha do tipo de parto. Investig Enferm Imagen Desarr. 2020;21(2). https://doi.org/10.11144/Javeriana.ie21-2.aget 